\title{
A Live Wire : Machismo of a Distant Surface
}

Marvin E. Kirsh 1

Kirsh2152000@yahoo.com

$\underline{\text { mkirsh@ calstatela.edu }}$

1 Department of Anthropology California State University Los Angeles

\begin{abstract}
:
The scientific study of socio-cultural phenomenon requires a translocation of topics elaborated from the social perspective of the individual to a rationally ordered rendition of processes suitable for comprehension from a scientific perspective. Scholarly curiosity seeded from exposure in the natural setting to economic, political, socio-cultural, evolutionary, processes dictates that study of the self, should be a science with a necessary place in the body of world literatures; yet it has proven difficult to find a perspective to contain discussions of topics in a coherent manner for scientific approach: for example, anthropology, the study of mankind, finds difficulty elaborating definition for the orientation of study; it is a member of the same set that contains it. In this presentation, based on features indigenous to a supposed distant perspective that is exposed employing experiences of history and criteria of common sensory perception, it is conjectured that a civilization lifetime pathology is contemporarily active. Example is taken from philosophical and sociological discourses, modern science theory, medicine in pursuit of international health issues, to capture conceptually a role of motions of external agents occurred within the interval of observation, elaboration of concepts, choice of directions, as a source of paradox and confusion. In supposition that does not escape simple logic, ubiquitously appealing to the experience bound senses for understanding, hidden motions, common to both observer and observed, are hypothesized to render from a sense of familiarity, a continued frustration in attaining an understanding of the self and nature. A psychical seduction is proposed to exist, related to historical behaviors associated with centrism and asceticism, produces eccentric interpretations that are bound modernly to logical circular, centric geometrical reasonings; world conceptualizations are conjectured to acquire an avoidance of a state of 'motionless' rather than death within selection processes. Projection by the imagination upon the unknown is conjectured to result in a seduction by an active "live-wire" embodied to motions occurred to a distant surface.
\end{abstract}


Key words: asceticism, centrism, perspective in social and natural sciences, behavior and selection, seduction of the intellect and civilization pathology, surface of experience and practice, entanglement

\section{Introduction}

\section{Radar}

No one exactly knows

Exactly how clouds look in the sky

Or the shape of the mountains below them

Or the direction in which fish swim.

No one exactly knows.

The eye is jealous of whatever moves

And the heart

Is too far buried in the sand

To tell..........

(Spicer, 2008)

Transcriptions of the discourses of the ancient philosophers Socrates and Protagoras argue the center position in the topic of knack and virtue to either men or nature: Protagoras, a sophist regarding the ability of rhetoric is reported to hold:

"Man is the measure of all things" (Poster, 2005) 
Protagoras's dialogue, appears to mean that men are free to construct their own ethics as if their activities molded nature and not in reverse; an unresolved related course of notions has emerged into modern day that is perplexed to find a perspective that elaborates satisfactorily with respect to nature.. The path of ideas through history to present day appears to emerge into two distinct modern philosophical presences from non-parallel trails. Socrates position, that men are but a reflection of nature, can be associated with turmoil and suffering occurred during the events of history. Socrates, himself was executed by a jury trial associating him with slefserving Sophist rhetoric, as a threat to the state. During these same times Jesus Christ and many of his followers fell to crucifixion at the hands of the Roman Empire. Proceeding through the times of the renaissance, the holders of newer world views, important philosophers, inventors, and church figures fell to criminal suppression and/or death at the hand of contemporary struggles to contain order within a chaos possessed to the many open possibilities entailed to a further explored world, more open, less containing philosophical outlooks.

Even as newer physical evidences inherent to a rapidly evolved exploration of the miniature, the cosmos, and the nature of the traveled spherical surface of the earth surfaced with reconsidered philosophical spaces; evolving towards modern world views, the paths of reason in discourses reflect narrower perspective divided between focus on the physically witnessed and a virtual constructed from the imagination that groups the unwitnessed or unwitnessible to the category "infinite" (Kirsh, $2011 \mathrm{a}, \mathrm{b})$.

\section{Discussion}

Centric views holding men and the earth at the center of the universe had been supposed chased towards the less open environment of the casket in a mortuary of regretted ideas, inherently amoral and cruel actions, and simultaneously supplanted with notions assumed to breathe life to paradox burdened cosmologies. Contemporary struggle today, applying the same repetitive behavior associating 'dead', though only absent cognitions, newly found selfperceptions intended to supplant the old, disappointingly reflect a long wished for, but only detoured with additional impulse, new openness The creation of real parallels, visualized in analogy to the branches of a tree, arising from a single seed, necessarily non-parallel, to establish a verisimilitude, truthfulness, are perennially observed to include logical accounts involving a bend-inciting 'infinity' upon the course of actions as they proceed from the past (Kirsh,2009, 2011a,b). From new awareness's intended for practice, made to the contours of demands imposed from the self-defeating natures of centrism and asceticism, there appears to have emerged from the willful sequestration of less sound concepts, a fatal self-infliction that can be characterized to bear an association with contemporary wide spread autoimmune deficiencies - Perhaps assessment of real problems are not complete; resolutions asking the individual to adopt to his range of awarenesses the products of inductive reasoning fitted from empirical test that employ sensory prosthetics such as microscopes, high powered telescopes, electronic imaging devices, to define the real are potentially more confining than therapeutic, even with accounting that involves increased population sizes for explanation.. Einstein (2005) elaborated a theory of relations attributing a capability of concepts to render either force or material substance (e.g. mass to matter in a gravitational field, or energy to matter, the bending of light 
when it witnesses mass), projections from proximal experience in application to a distal unwitnessible that is embodied with the creation of a lifeless out 'of the world' zone, a bending of concepts as the means for the elaboration of substance. Einstein's notions are not only self contained to the premise that the living/viable and lifeless are divided among separate spaces and distinct in root character, but, as many, though not all (Foucault, 1977), arguments have evolved within the history of ideas, is detoured from an absurdum reductum inference that entails a force possessing energy to conceptual processes, excludes the conceptual from nature.

Modern anthropological (or archeological) theory becomes logically frustrated for definition that does not include elaborated aspects of the interlocutor; has come to refuse description that does not reference the consequence of interactions that necessarily involve the persistence of the past within all elements and interaction of those elements; the archeologist has found it compelling to treat inanimate material products of past cultures as 'things' with a living history.

Is there something perverse, if not archly insistent, about complicating things with theory? Do we really need anything like thing theory the way we need narrative theory or cultural theory, queer theory or discourse theory? Why not let things alone? Let them rest somewhere else-in the balmy elsewhere beyond theory. From there, they might offer us dry ground above those swirling accounts of the subject, some place of origin unmediated by the sign, some stable alternative to the instabilities and uncertainties, the ambiguities and anxieties, forever fetishized by theory. Something warm, then, that relieves us from the chill of dogged ideation, something concrete that relieves us from unnecessary abstraction. ((Brown, 2001, p.1).

From frustrating attempts at analogy of cultural evolution to biological evolution, theory in anthropology has drifted away the employment of scientific like constants and universals, to focus descriptions on intervals that occupy describable spaces situated somewhere between what would normally be construed conceptually as beginnings and ends in parallel to the birth and death of individuals. It is possibly, though, the case that all processes proceed in a recurring manner in a direction of death towards life, rather than the reverse, and are funded structurally from a "conceptual energy" that is born by analogy from the physical confrontation of spaces upon encounter: the universe is more accurately described as processes of inheritance and change other than as a space containing processes, a genetic process of proximity based inheritance maintains the character of past encounters within an unfolding that necessarily finds new points of navigation if the birth of the unique identity is its only content, otherwise to refer to a null state that is both still for description but sufficient to describe motion in fantasied intersection with it, including no accounting of action/reaction, momentum acquired from encounter, logically coherent only if 'discourse' seen unique to human cognition is reconsidered to mean interaction, force possessed only to gravity bearing nominal; the mind, if it can effect change, in the process of lending description endows forces belonging to the same category of forces and actions endowed to objects in motion. It might be logically sound to infer that the universe is not divisible into distinct spaces of contrasting character. Einstein seems to have creatively imagined a world with parts that are immiscible with the self in allusion to a 'concept' 
that becomes infinitely bent at its lifeless limits, though existing still ubiquitously as mass, describing some spaces to encompass, as if an environment in tumultuous change without enduring features, occupying infinite arcs within the fields of vision of relating witnesses, a hidden nature of the world that is absolutely beyond witness.

If a mathematical rendition of nature is not believed possible, at the extreme, the universe can be constructed from description that is devoid of restrictions imposed by the confines of the products of logical and rational interpretation; description assembled from logic combined with language, as the feasibility of some mathematical description becomes apparent, presents a quagmire to the imagination in attempts to construe only parallels to construct conceptually, excluding the existence of the nature of 'the concept' itself, concepts are seen to evolve to entail physical parallels within nature. In this presentation only the conceptual bear description possessing the quality "parallel", the physical is restricted to physically connected non-parallels. Perhaps relativity theory should reserve discussion of the path of unwitnessible, unreflected for witness, light energy to the conceptualization of a concept of "the (change effected/effecting) concept", a conceptual that is made strictly of invisible differences embodied to a concept bearing, proximity dependent for function, nature other than to position/acceleration based differences in rates of energy metabolism. Conceptual construction is the only means of rendering theory that does not contain visualized particulars, e.g. of location or dimension, is truly universal. Proceeding tests of the theory of relativity (Ouellette, 2011) employing satellites armed with gyroscopes able to fix positions in space very precisely, intended to measure distortions of time-space, requiring years to interpret employing high speed computers, in their conception perhaps physically mirror or imitate the projected nature of a situation itself that has not been conceptually characterized adequately; the discussed complexities of the extended projection of theoretical and empirical lines by intuition and induction suggest that extracted data may refer accurately not to a warp of space (space-time) but to a change in measured parameters occurring as a consequence of a change in the relationship of uniquely engaged conceptually parallel identities-e.g. earth/civilization and star, IM Pegasi-concepts in theory employed in interpretation are ubiquitously infiltrated with "physical-mathematical bend "ideas, are space-reducing if measurement interpretation is made with analogy to an 'infinite-bend beyond-the-senses-null-state possessing the 'beyond-the-experience-able-world' concept, 'mass'. 'Mass' as a value is better accommodated to refer to number within the paradoxes and unknowns encountered in efforts to elaborate a rationally orderly cosmology containing both number and nominal.

From this perspective space is overly occupied with space-filling numbers. It is perhaps logical to imply that modern interpretations entail an attenuation of, rather than growth of witness relations (e.g. the world is expanding away from the earth) in a self-determining manner towards death, death of associations. In actuality, other than a set of location-less characteristics of specific materials, it is the unique path of emergence of the identity of each entity that renders its' properties, ideas of parametric location and emerging identity are modernly confused during study and maintained conceptually to a situation that renders a compatibility with scientific method and is adjusted to compensate for a lack of understanding of mind and matter in association; though construed ubiquitous temporally long physical paths of emergence of processes are unconditionally beyond witness, they cannot be elaborated by the imagination to be of different character than the proximal at hand. In a universe framed with respect to 
engagements, other than the pure elements that compose entities, it is the unique emerged composite of elements that define an identity and can be referred to as location-less to possess within it traits whose measurement entail the study of elements that have origins that are tangential to the line of interaction, are not containable to the same frame as the relations they effect. Supposition suggests that the red shift employed to establish distances of celestial objects (de Sitter, 1934) refers to motions relative to bodies other than the earth. In popular culture, even among a few scientists (Connor, 2008, Pravda, 2010, Fox News, 2007), as well as in science fiction story, or in ancient myth, ideas exist, whether believed to be realizable or not, of (a need for) time or space travel that is effected by physical, conceptual projection of the self to new locations; of antigravity devices, of the ability to bend matter with energy from the mind, of astrologists giving future predictions: a sought autonomy of men to take charge from a nature that births them is willed in an accelerated progression towards death, rests upon a lack of conceptual awareness of the motion of the observer, motion inherent to all observation and the material components, internal and external, that consequent it. If nature can be contained in definition to the category of motion it not logically coherent that it is possible to both assume command of transmitted natural impulse and exist in all aspects of being as the product of their reception/transmission, as pushy behaving, enroute to conceptual status, unmoved movers short changing themselves of naturally granted spaces.

History modulated workings that bring about the present and the future, in current perspectives, would necessarily accrue additional acceleration realized to attenuate/expend lifetime energies ensued from a universal/constant based natural philosophy in a manner analogous to damage visualized occurred from impulse applied to a waterway perceived to have rigid walls though they are flexible; induced space-time notions possess an incoherency and ambiguity; rather than shared time attributed as a single parametric value within entities engaged in witness relations, duplications of occurred time to individuals is manifest to descriptions that account for it distinctly on an individual basis, in a one, verses two, at a time manner; the passage of time to a witness engaged in relations has no means to witnessibility by the other agent and cannot be said to be quantifiable; delays in transmission, reception of signals can be attributed only to transit times of energy in the establishing of contact between transmission-reception devices and have no relationship to the passage of time, rates of processes at different spatial positions: invalid philosophies effect an amplification to the relative motion of observers that detracts from system tallies, to compress space, reduce lifetime time upon application. Changes occurred with time between identities are of a different nature and source than those attributed to the composing elements of particular identities. As an example, though fingerprints used to identify are unique to individuals, are necessarily unique themselves and cannot be extracted referring to recombination dependent diversities, but are the products of the same uniqueness of spaces that orchestrate unique genotypes; phenotypes : the descent of energy to acquired form follows only a path towards its physical localization to matter as a condition towards the accomplishment of contained order, identity. Other than cosmologies containing mass, energy, time and matter a better cosmology is conceived to possess only number and the propagation of force to render identity possessing form.

As an entailment of relations in a perspective that is absent of the general imagined condition, it is possible to think of triangulations that are conceptual only in the example that has three components (witnesses in witness pairs and a universal perspective that involves a null condition, 
velocity $=0$ ), or alternately in explanation to conceive a physical triangulation involving a witness pair representing two vertices and an unknown physical entity/identity to represent a third vertex employed as a non-universal perspective. In this presentation, other than a null state, a third surface, existing ubiquitously to environmental relations, within the self , not discriminated distinctly by the cognition, is held responsible for misinterpretations of nature, defeating behaviors. From this perspective, time travel, change willed to the past can be referred to a change inducing contour of a distant or hidden surface that is active over vast time periods, conceptual energy of the mind acting as a lever might conceivably bend matter if force caused by the described pathologically associated attenuation of the size of spaces can be attached to exist to an long term existing environmental pathology, the future might be predictable to the extent that a chronic and malleable influence has been active over long periods. It is important to note concerning recent recreational products or conveniences in which the skin with attached electrodes moves virtual objects on a computer screen, or activates servo motors, that forces involved maybe imitate or are the product of a threat to the integrity of an unknown agent to result in the perception and conception of mass/number rather than matter to its' composition; their employment maybe pathologically destructive to the integrity of the individual. Likewise, high energy experiments to create new heavy elements may contribute to offset naturally arrived balances and orders.

In the process of elaborations, of self and notions of environments, employing scantily understood relations of body and mind, construction endeavors overlook a world confined strictly to structural and functional description employing ratios, the possibility that both the pathological and non-pathological are the strict consequence of the processes of contrasting surfaces, the world more labile than experienced, is not recognized as concepts are given physical room on drawing boards. Protagoras in his dialogue with Socrates, might had meant that man, rather than an example of nature, is an example of those parts of nature that pertain solely to man; the case is argued for other than an infinite universe and finite man, or infinite universe and infinite man, for a finite universe and finite man, to suggest perhaps that confusions entail in addition to a failure to acknowledge that men do not represent or experience all of nature, they are confined to define knowledge to what is strictly emanated from the witness relation man/nature that potentially bears a deception, the consequence of the existence of a third surface that is present physically and conceptually Nietzsche (Nietzsche, 1967) believed more than two centuries ago that a weakness existed that was possibly of a medical nature: he commented at the time that men thought that they had conquered themselves; from the described perspective, confused over , mind and matter, themselves, nature, the internal and external, it appears that they may actually had successfully won conquest physically, militarily in battle with themselves in a contest meant to be strictly conceptual in nature.

Willfully blended character traits of the empty null and of that which exists, involving alien/mathematical spaces unable to support life in an infinite universe disguise the potential existence of a third party, that, other than infinite, is both familiar for knowing and, deceptively in analogy to an infinite that is construed to exist without visualized substance, is invisible to the senses. Modern discourses, evolved to discount, based on physical revelation, ideas of the centric, 'centric man' existing in a world centered around him, include mathematical description, might, in a perspective that construes motionless and death together, though objects of observation are necessarily in motion , chase engaged witnesses, in a path that is otherwise 
than a best energy, high efficiency, open, linear transmission of contained form, from birth towards an unreachable, infinitely bent outer realm, premature death: the mind drawn to construct dualities; coexisting perspectives of a rationalized nature, from self -denial, that is ascetic rather than fulfilling, alluding to refer continually beyond the elements of realisitic frames of attention (i.e. space, the social, political, economic), is never able to capture them together to form viable parallels of one to the other that do not triangulate in threesomes to a far undefinable beyond, in actuality referring ultimately to a pathology residing beneath the surface of the skin and acquired from processes that more proximal that a supposed standard of infinity. . Modern views in physics possess paradox that is bridged inductively with ideas of length contraction/expansion, tensors, is necessarily determined unconquerable by the nature of the, self-defined elements employed for the definition of perspective. Lorenz transformations (Zeeman,1964, Einstein, 2000), an expanding universe defined from theory and observation that celestial objects moving in an outward direction obey a mathematical law in description of rates of motion (Field, 1988); pointing in the opposite direction of the observer logically ensued is the description of a threat to identity, diminishment in the wake of imagined replenished/increasing distances of the self to the external in a cosmology that construes distance to be infinitely abundant, inexhaustible. A self punishment threads its way through discourse from ancient times.

Thoughts that men and earth are universal centers, presumed buried, are yet immutable and indestructible as are all concepts; especially as concepts bearing a sufficient lifetime to have accrued an accountable temporal path, are but parameters inherent to a path that necessarily ensues from path, are invisible elements within a setting established to appear otherwise than man-centric. It is from this description that evolved cosmology is suggested to bear a descriptive empirical, rather than conceptual foundation. Cosmology, defined as the branch of philosophy dealing with the origin and general structure of the universe, with its parts, elements, and laws, and especially with such of its characteristics as space, time, causality, and freedom (http://dictionary.reference.com/browse/cosmology) cannot contain physical renditions of an empirical nature, but of the nature of 'path' itself as it is able to contain, rather than describe, experience. A historical, political-social, association existing to cosmological discussions witnesses a slow to resolution, potent power on behavior that is likewise prominent in temporal content that is prominently aware to the perception, rather than concealed to the labor of the unwitnessible conceptual energies of the mind for expression.. Framed within this discussion, it is conjectured that ancient and modern writings, perhaps biased to penetrate more deeply into visible geometrical form which modern science is supposed evolved, are ultimately biased without reference to a nature of 'the concept', theorizing the nature of a reality that is restricted to the evidence of material substance, ensues to passenger yet further perplexity when objects in theory exceeds the common senses, from which modern method to induce intermediates in the investigation of physical action with imposed physical action, trails with the "idea" that the idea has a biochemical nature, yet untried is the idea that the physical nature of thought and a nature of the physical are intimately intertwined. The ancient Greeks might had been flattered, impressed with the thought that the elements of the world had a consciousness liken-able to their own, but it is possible that the same reaction might not emerge in a contemporary settings considering paths taken in civilization frustrations with physical nature, the emerged state and problems of modern societies, though a potentially less complex, sound to the senses outlook may lend valid relief from attributed excesses to possibilities of structure and function attached to nature, what is knowable and pertinent.. 
Frustrations towards the solutions to problems lended more and more complex daily by observation, experiment and practice is apparent, for example, in the same manner that the AIDS virus has surfaced world wide at the exact same locations where the smallpox infection was eradicated employing a living vaccine (Weinstein, R. et al, 2010, Halverson,2007, ) though the potent factor in AIDS infection is also reported to be susceptible to the small pox vaccine, a common frustration always remains to endeavors, emerged spaces are occupied deleteriously, embody a struggle of the self punishing itself and the egocentric self, asceticism and centrism, each elaborating the other. The case of AIDS and smallpox reflects a failure to establish a realistic place for the self; evolved (in a smallened world threatened with autoimmune disease) spaces instead are occupied with pathological processes. Perhaps relationships between individuals in efforts to contain the self realistically and instinctual reflex that makes the self the defining spectator of the world, have more mystery than is apparent to surfaces.

In the process of this elaboration, an elaboration from description into a sought logical order of processes is attempted that employs analogy to common experience in which concepts are redeemed to propose the existence of a live-wire; human nature redeems instances of experience, modernly trending to concede a more relative nature to the path of processes, human processesfacets of capitalism, the redemption of work labors for wages that are redeemed for goods and services, the architect drafts with pencil and paper drawings realized to actual buildings, the archeologist, unable to realistically characterize the individual contribution in the production of material culture, hoping to elaborate a clearer meaning to human engagements from material artifacts of the past attempts to confine conceptually social processes with analogy to contemporary life, activities and experiences, considers formulating the world in terms of the life of 'things' that maybe either living or inert (Brown, 2001) a socio-cultural cosmology, natural cosmology situated from within the sphere of contemporary observation and experience, based on the familiarity of surfaces, living to living, living to inert, the ethnographer in modern trends redeems of the self, experience for what has been acquired in studies, to write literary oriented description hoping to reveal shadows of culture to the perception and cognition.. The world has come to be realized to be unrealizable to the tangible effect of processes beginning at point $\mathrm{A}$ and ending at point $\mathrm{B}$, nor can it be realized to be entirely relative to lose definition, it is a process of setting dependent social participation, redemption that has no other defining boundaries. The redemption of conceptual assets mandatorily acquired from test or experience/practice into capital assets appears to be a single innate process, might suffice to describe all processes. A real potential to redeem frustrations and unresolved paradox for a self-granted license to supersede naturally found mechanisms can be frightening considering the potential absence of standards in fields of pursuit that philosophically are strictly relative: absent of natural homeostatic mechanisms, if the quality of 'familiarity' is not first applied to guide pursuits and frame a stable standard of reference amidst a necessarily dynamic and fluxing, 'familiarity' redeeming universe that is, logically cannot be, entirely present to witness.

Evolved from inductive reasoning, compared for validity via test with the empirical, much of modern day science theory is realized in practice as technology applied towards the efficient utilization of natural energy for the resolution of the socio-cultural problems of an expanding global population; products of the imagination are redeemed, employing natural resources in a device to provide certainty and physical comfort, imagined form and function are seduced from a locus in the experience guided imagination to a physical location, the past is redeemed to 
produce a future, a path from past to future, essentially determined by cognitive processes whose etiology is arisen in a mechanism involving proximal communications in a haphazard appearing cultural evolution that does not seem interpretable from an extended perspective. Revelation seeking experiment modernly reaches deeper from the proximal niche inwards towards the interior of the cell, the earth, outwards towards outer space with a vast investment of assets, conceptual or material. The revelation of truth has become deeply entangled with a wishful process involving the physical exploration of locations that are infinitely beyond immediate or extended experience; survival progress has come to be entailed to the exploration of unknown locations employing a process of cognitive projection of familiar aspects seen to be most essential of the self to unknown environments in advance of their exploration, at the extreme, a wish to redeem struggles with a physical teleportation of the self. Employing the same assemblies realized from accumulated experience and imagination, in analogy to a stage play with created characters given life from the internal mental experience of actors, the value 'distance' is buried within schismatic anthropomorphic realizations of an ability to engage any or all locations, on one hand as if 'distance is distance', all that exists are places divided from another by 'distance' that bears no parametric description; on the other hand, distance that is in a mirrored manner, parametric in nature at the distant location. The features of Mars are sought to determine, employing imagery, whether they can support life: 'Let us suppose", irrationally (it is not philosophically sound to assume that Mars would be a viable venue), "that our conversation is on the surface of Mars". The products of human activities similarly occurred can proceed in a continuing series; if it can be imagined that the number of human events, paths to actions accumulated from a particular point in the past to the present instant can be captured to a finite number that is entangled to the aging/change occurred to all things dwelling within an associated radius to events and does not represent all possibilities, it might be argued similarly that if the number of imageries is environmental-contour dependent, continued setting to setting, is similarly finite and less than the total possible for all dwelling possibilities, either (actual events or imageries) might be captured to a closed surface for a given interval whether the interval represents a whole species lifetime or less. In an extended analogy (for instance the number of squirrels verses number of mammals instead of occurred events verses imageries) it might be inferred that it is possible to capture total numbers for these interdependent related genera to unequal finite surfaces parameters, parameters evolved from within inherited states of imagination and induction, and those evolved from within prior states by natural mechanisms of proximal inheritance. If conceptual produces universally exceed in number material produces, temporal periods to finite closure, i.e. death of spaces, might be reduced if the conceptual is redeemed for spaces that are not contiguous with the emerging spaces mutually inhabited by conceptual and material facets entangled with that from which they emerged. A conservation of distance, as either conceptual or physical energy is expended, seems, as a rule, to conserve/strengthen identity from physically expanding, artifactually produced diffusion , occurrences, that weaken identity and shorten lifetime by effecting a premature closure of surfaces. Framed with respect to the process of diffusion, birth, an act of containment, and death an act of dispersement, are diametrically opposed to one another, as in common sense, though the world might be comprehended more satisfactorily, thermodynamically more understandable, if it is viewed not as having been born but as processes funded by the conversion of energy to matter, the conversion of vast distance to small distance, proceeding from dispersed state to contained state. Life, cognition, diffused, by proximal inheritance into existence, can be undone 
if energy to entities is invested to effect 'distal' rather than 'proximal' transmissions of acquired organization. Men are able to function as either creators or destructors of material substance; as efficiently as they are able to fertilize eggs with their own sperm, invivo, they are able to fertilize them in a laboratory, clone genes and reinsert them into species, plan the cloning of whole organisms, entangle together virtual, imagined activities and their imagined material products into self-made virtual worlds within which real global economics, bank transfers fund virtual activities.

Obviously non-essential to the survival of species endowed with lesser ability to visualize long chains of cause and effect, the process of 'teleportation' of the conceptual elaboration to distal material existence, appears to be an innate and natural capacity restricted to Homo sapiens. Fantasy applied in construction to test the physical can be construed to be common during development, lower animals are seen to act in play that has no apparent immediate survival value. Home sapiens with cognitive abilities, the youngest of the species appears in the tree of evolution at a division of the non-effecting and effecting, he is able to conceptualize and experiment either cognitively and/or physically with the element of contingency, at the same time failing to admit a unidirectional nature to the element of time as natural processes accumulate material structures, things, that necessarily have an immutable history. A world visualized and framed uniquely as a set of interactions in which all elements can be accounted for and dissected inclusively with respect to mirroring, symbolism, and need (Malinowski, 1972), time, an artifactually made concept referring simultaneously to experienced/witnessed motion and path, excluded as a basic element, does not provided for imageries that can effect willed change to change that has arisen naturally, as described by proximally propagated motions, mechanisms of proximal inheritance from within.

Unaccounted in this description, is seduced and nasty behaving mankind, perhaps reacting to necessarily either self or naturally instigated behaviors comprising an extensive list. Sound interpretation refers behaviors to a nasty medical pathology, nasty and meticulously contrived David-on-Goliath behaviors in response to a nasty unrecognized infection, suspiciously, rapidly emerging temporally to involve the military, scientific and medical armed with philosophical misconceptions, appearing propagated since the first recorded discourses regarding the nature of the world.

Modern terrorist as well as military activities range from the delivering of military arms vast distances with unimaginable precision, contrivance, using cloning techniques devised in laboratories, to induce disease in military opponents and populations, through the postal mail, unmanned armed drone vehicles operated from great distances, spying operations employing satellites that find no obstacle in the curvature of the earth, time and distance differentials. Medical applications have come to rely less on naturally created remedies, but artifactually produced molecules from microscopic studies of the contours of the micro miniature and similarly effected rearrangements to genetic materials to cause change that sometimes witnesses difficult pathologies rather cure; motivations oriented simply enhance agricultural production yields can cause irreparable ecological damage. In different perspective, appearing to border on pseudo science but with strict objectivity in mind that is centered the witnessible features of occurrences, all of these behaviors might be viewed as an uncontained living out of experiences with a nature that has not been contained philosophically, as if within an accident whose 
evidences are not all sufficiently apparent to stimulate the imagination or belief. The eradication of smallpox brought about the unforeseen surfacing of auto-immune deficiency syndrome (AIDS) whose mechanism has been discovered to be intimately entangled with that of smallpox (see above). A list of pathological features of smallpox and AIDS might include not only the artifactual subcutaneous invasion of live virus to combat infection, but some sort of naturally effected temporally occurring juxta-positioning of genetic elements as well as a temporal disturbance of perception effected conceptually in understanding and ensued behavior: an unknown occupation subcutaneously beneath the skin of all that is within physical grasp and familiar to sensory perception is simultaneous with concept formation. Visions of a nature instinctively known to be tree-shaped are distorted to see trees as but a part of a nature that reaches toward the open of space that has some other shape; proximal/distal, distance/time conceptual rearrangements appear to act during more the reflective, reconsidering moments of analysis-if large trees take up too much space for purposes they are made smaller in order to effect arrangements perceived harmonious to a proximal composed of small distances and time periods supposed universal if observations add to equal the reality and dimensions of theory...

The living out of the proceeds of a pathology is expressed in eccentric behavior that occupies a greater arc in the field of vision, more time in actions, but exist normally among a total of possible things men might do with matter. The genetic tampering, in a subcutaneously manner with trees, distorting their size, physical range of branches, on further reconsideration can be seen as the invention of a means of physical support of the trunk by the branches within the progression of denial of an occurred or pending fall from the tree.. A long list of deleterious behaviors can be assembled.in which plausible interpretation entails the force of gravity upon a surface that operates unperceptively over vast temporal periods with respect to the human lifetime; or exists undetected entangled to other places though its' features are not beyond the capabilities of modern science technology made aware of bio-sociological aspects of problems. Physical evidence existing for the impact, both past and present, of many objects with the earths' surface, objects within reach in space, might lend a reality to possibilities that false interpretation of nature in the construction of cosmologies has occurred. The set of natural processes, in which man is both product and producer, does not in reality make distinction between expanding and reducing occurrences, but that all facets not only proceed in, but are contained to, a tree shape path of space.

Renditions of entangled states in physics theory (Bub, J., 2010) seem to accommodate the progression of nature through trait retaining branched paths but fail to distinguish an anthropomorphism necessarily inherent to human producers, the world is confined to begin and end within sensory experience, the only possible major division coherently attachable to nature is a disparity of sexual gender, the world functions by sexual reproduction, the rearing of offspring, from which knowledge of a nature that births the new from disparity occurs. It is possibly claimed that gender exists apriorily to all of nature, takes priority to all, is not in a scientific sense evolved as if it suddenly appeared in living entities; interpretation is logically confined physical divisions bearing potential differences, but in a familiar, whole and indivisible sense the world is dividable only with respect to human gender, male/female, disparate cultural processes that bear sexual gender disparities to their sources. In his discussions of entanglement theory and the teleportation of traits across distances Schroedinger, referred the source of 
'character' to 'interaction', he expressed a certain discomfort with the idea that change could be caused at a inaccessible distance.

Another way of expressing the peculiar situation is: the best possible knowledge of a whole does not necessarily include the best possible knowledge of all its parts, even though they may be entirely separate and therefore virtually capable of being 'best possibly known,' i.e., of possessing, each of them, a representative of its own. The lack of knowledge is by no means due to the interaction being insufficiently known - at least not in the way that it could possibly be known more completely - it is due to the interaction itself...... It is rather discomforting that the theory should allow a system to be steered or piloted into one or the other type of state at the experimenter's mercy in spite of his having no access to it. (Schroedinger,1935).

Bub writes: He (Schroedinger) found this conclusion sufficiently unsettling to suggest that the entanglement between two separating systems would persist only for distances small enough that the time taken by light to travel from one system to the other could be neglected, compared with the characteristic time periods associated with other changes in the composite system.

Until John Bell, 30 years later, falsified Schroedinger's notion that entanglement decayed with time, established that entanglement can persist over long distances, physicists supposed explanation to Einstein, who claimed that a common cause was responsible for behaviors, his inclusion of the neutral observer.

In original perspectives, lacking other explanation, action at a distance, considered philosophically absurd, requires the existence of an ether through which light bearing time/energy must necessarily be propagated. It seems simpler to make analogy of Schroedingers' 'representative' with 'the concept'; born from interaction, 'the concept' bearing past-experience dependent form in its' bearer, in a tree shaped manner upon disparate concepts (representatives) forms meaning in language that can be changed spontaneously in latter-branch engagements with the insertion of different/new meaning:

Suppose Alice and Bob share an entangled state of the sort considered by Bell, say two photons in an entangled state of polarization. That is, Alice has in her possession one of the entangled photons, and Bob the other. Suppose that Alice has an additional photon in an unknown state of polarization $|u\rangle$, where the notation ' $\mid>$ ' denotes a quantum state. It is possible for Alice to perform an operation on the two photons in her possession that will transform Bob's photon into one of four states, depending on the four possible (random) outcomes of Alice's operation: either the state $|u\rangle$, or a state that is related to $\mid u>$ in a definite way. Alice's operation entangles the two photons in her possession, and disentangles Bob's photon, steering it into a state $\left|u^{*}\right\rangle$. After Alice communicates the outcome of her operation to Bob, Bob knows either that $\left|u^{*}\right\rangle=|u\rangle$, or how to transform $\mid u^{*}>$ to $\mid u>$ by a local operation. This phenomenon is known as 'quantum teleportation.(Bub, $X X X)$

In the search for universal models, it is logically more coherent to frame them with respect to criteria of container/contents only, familiarity as the exclusive basis of interaction: additional components, such as time, distance, and ether in basic description are not warranted, lead to theory incoherencies: the attribution of memory as a universal trait of all spaces, specifics attributable to tree-shaped confined paths in a trail of engagements/interactions, the indelible 
product of encounters bearing the same, bears no null condition or neutral observer and entails that a lack of observation, hence lack of interaction can have no coherent meaning for model production. In the above example of Alice and Bob, a common cause that cannot be disentangled from a descent of meaning from a common containing element possessing an appropriate single representative of both Alice and Bob, is proposed to be the case. It is reasonable to hold that the existence of paths of unreflected and hence unwitnessed energy might be as far as inductive reasoning can be extended: their witnessible products are mutually entangled with a dynamically changing unwitnessible whole to be in some way mutually related in a manner that inquiry with respect to the existence of parallel paths strictly refers to a conceptual that is born of differences; an extractable nature of physical relations of consequential reflections causing witnessible change can be only of a ratio nature, witnessible line $\mathrm{A}$ yields witnessible line $\mathrm{C}$ by comparison with witnessible line B. A universe possessing straight, hence physically parallel lines entails all lines to be parallel, disentangled, other than a hypothetical, the existence of only a null condition. A tempting analogy is possible in light of discussions that are completely engrossed with unobservables and invisibles:

'Well every time you and daddy brought home a baby, you wouldn't tell me where it came from so I assumed something else put it there, how does it look like, me or mommy or daddy and not Jesus Christ.'We all, like Jesus Christ might be sacrificing more than we are building, but no one can profess to have seen him for themselves; because each mind with its own distinct space composes only from disparity and difference. While it must be that an encounter has occurred while I was not present, there is no possibility of an existence that is ubiquitously absent.'

As in the above analogy, an anthropomorphic quality of entanglement is not only possible, but observation and experience is strictly limited to the acquisition of character from within; from the perspective of human observation witness to containing elements is not possible, the induction of aspects acquired from imaginations of possible encounter are capable to mislead the mind to endeavor in that direction. Cognition, language cannot be modeled to be a mirrored counterpart of physical nature as if it is no more than the product of association with it to bear a representative of it, to bear it as a representative of a null lifeless condition, in converse, such induced models bearing induced representations of human nature may indicate a positive trend to seek meaning to associations that is more aligned to the individual in a similar manner as the shape of constellations bore anthropomorphic design in ancient cosmologies. Perhaps original renditions to bind anthropological science to biological processes (Malinowski XXX) with criteria of mirroring, symbolism and need has broader universal implications than originally imagined.

Entanglement theory has, since the 80's, taken directions armed with the philosophy to exploit phenomenon for study gains, headed with the phrase "The Joy (of)Entanglement" (Popescu and Rohrlich, 1998) suggesting a potential to augment conceptual spaces employing forces of great physical leverage upon (conceptual) volume-bearing states of nature, a great danger to occur a mutual damage of both a physical and conceptual nature is entailed, engagement with black holes, the projected object of experiments, proposed, probably correctly, to possess internally gross distortions in energy/matter processes, internal states, cannot but, with aggressive encounter, become entangled with observation/natural states of the observer. 
Alluring/seductive, other than joy, abandon rapture approaching enduring disorganization, is suggested.

A near perfect mathematical rendition and associated philosophy of the shape of an egg has been produced (Kirsh, 2011a). From very simple mathematical relations for the construction of distances occurred to the progression of a sphere along a line (though graphing points do not result to be adjacent to one another as needed for direct calculations of surface parameters-as one might expect models of a real nature to behave), it might be feasible to engineer physical models for the evaluation of parameters related to the maximization of conceptual lifetimes entailed to the interaction of physical spaces.

In alternative to closing this inquiry with the presented theoretical considerations, it is appropriate to present physical analogy that maybe profound in less extraordinary ordinary examples. All mammals test the world with experiment that is guided instinctually; a dog playing chasing a ball naturally chases things not intended for prey in daily activities, a chimpanzee imitating behavior may grab a magazine, sit down in front of a TV, turn the pages pretending to read it, or unknowingly avert diseases with repetitive behavior that appears to be passed down to off spring by simple witness. Behavior to exploit vast reserves of petroleum from which modern transportation depends as well as most of the materials of modern society depend for their production, may reflect but a willingness to profit from an entanglement of the earth to processes that are the probable consequence of impulse imposed during the course of natural history externally upon the earths' environment by an unknown surface. It is possible to imagine that externally arrived impulse occurred upon environmental elements can cause the individual subliminal pain, result in poor food and air quality. The imitation, by habit, of a pain evoking motion of nature can produce addictive behavioral tendencies as the direct reflection of the nature of an enduring long term natural trouble that is secondly emerged from habit as addiction. The use of addictive drugs is a world wide problem, involves vast amounts of natural resources, currency flow. It is interesting to note that cocaine is harvested in less technologically progressed world areas, transported and used elsewhere where the suggested stage of imitation of nature with the application of impulse to it and progression of exploration associated technology partner one another. It is logical to infer that phenomenon divided for understanding into the longitudinal (from the past) and transverse (contemporary areas, volumes, regions), such as for the interpretation of cultural phenomenon, the dispersion of myth, etc., in actuality possess but one longitudinal element that is entangled to a unique past event, the forwards arrow of time has no application in the description of physical distance between loci unless they are mutually influenced by a single temporal element. An appropriate perspective for real familiarities and hence a conceptual unity to phenomenon is failed to be resolved; in the sciences simultaneity of time cannot be discussed unless a common physical element exists simultaneously to appropriate witnesses (Kirsh 2011a,b).

If imitation behavior is considered to be atemporal in nature, represented in all intercourses, addictive behavior is not necessarily simultaneous with it or spontaneously acquired in Homo Sapiens. Occurrences of drug addiction might surface to be location dependent, from the interface of unknown loci external to cultures, drug addiction might had evolved from habitual behavior that re-creates subliminally experienced pain. If sociological problems can be envisioned to trace a path beyond the familiar to a single surface, the expressed intensity within 
a particular number of victims and/or elements resolved to be related to the expression of behavior problems might find correlation to distance from their earthly sources in properly sorted demographic data. The convenient marketing of drugs in mass media shows a commensurability of 'distance-less' in communications within media engagements that is compatible with the idea that an unknown distance lurking beyond view resides to instigate behavior that may embody universally in a perspective in which a tangible distance at locations of drug dealing and addiction is teleported electronically and given new existence location to location. Behavior transmitted via a world library, for instance, in which nuclear genes have become a topic of science for the free willed application of impulse to effect species alteration, might have a similar etiology underlined by habit, if not a wish to induce change; a new science and drug addiction problems with a like origin in the experience of subliminal pain originating from a common third location. Intuition suggests that such parallels, upon physical triangulation with appropriate criteria, in the same manner as myths can be found to have geographical/historical sources from which they are dispersed, though existing in actuality in end-to-end temporal/physical series with a surface that is beyond view to effect, envisioned from a contrast of series verses parallel geometries, a realizable long duration physical distortion causing both the reception and transmission of bends within nature, the mind, concepts and personalities-e.g. a subliminal pain that ubiquitously falls beneath the shadow of an external nominal cause is propagated with number/mass, rather than identity/name occupying the intellect.

It is noteworthy that tattooing behavior is spreading at an increasing rate in modern industrialized societies. Tattooing can be conjectured, at its' root, not to refer to the ubiquitous parameter of identity, expression of uniqueness, but to conceptual paradox emanated from ideations of identity-less/unknown numbers/masses verses unknown nominal identity; paradox of number verses name, in a field of a possibly assumed eternal occupation beneath the skin, or the existence of unknowns might be envisioned to be culturally dependent on the region specific role played by science, as discussed above with an ability, acquired by virtue of a blindness to the existence of external factors, to teleport in a process that revitalizes proximal dispersions of habitual behavior. Crowded regions in high technology settings may show for instanc high volumes of persons with tattoos, high numbers of tattoos, or large sizes of tattoos per person, or more tattoos with lettering and numbers. A spreading name-number conflict, threatening identity constructs, is postulated to triangulate physically both beneath the skin and towards unknown physical sources, towards an unknown surface as it engenders the channeling of economic resources that are invariably rationalized to proximal material and/or conceptual 'profit, but maybe reflect instead actively entangled states birthing conceptual witness via a voluntary proliferation that has at its' root unaware subliminal impulses. Unnecessary selfinfliction is witnessed in behavior as tattooing, drug addiction, auto-immune difficulty: many of the social, economic and environmental problems of capitalism, associated with powerfully drawing behavior within the cell, external to it, external to the individual might be attributed to conceptual habit related to a complicity of the individual with hidden motions of nature; experience that might be sufficiently confusing to account for observed problems.

Consider the recently used term "immergence" (defined as the processes of disappearing by entering into any medium, as the moon into the shadow of the sun http://dictionary.reference.com/browse/immergence) that is employed in discussion of social and genetic. Immergence is used to describe a temporal connection of the old and new to form 
synergies reminiscent of a self-guided free-floating self-elaborating bubble grown exclusively from the external environment that resemble research endeavors in physics based on gravitationless space concepts in the assembly of a self-support from the bootstraps (Yoshida and Saitoh, 2011, Zhukhovitskii,2006) that can suspend or levitate. The conceptual product of a timetraveling intercourse with the past, other than the generation of the varieties of the species, immergence is employed in descriptions that resemble the self-willed tampering with genetic structure to produce hybrids inherently different from those naturally emerged, enables the indulgence in research directions with a delusion of expanding capabilities for explanation. Martinez (Martinez,2007, 2008) has demonstrated the mind to be capable of reflexive recognition, assembly, disassembly, of meaning externally acquired by interaction and effected at very primordial processing levels, or to be able to lose sense of its' location when motions effecting sensory experience are mechanically entangled with cameras to virtual images made present simultaneously to vision.

Learning or survival by imitation is most likely very elemental in animals: if it is a deleterious product of human induction, is itself a product of motion-created/creating seductive impulse that is driven by instinct to imitate, produced within the elaboration of contingency originated environmentally; a scientist does not need for his own survival to mediate change, his will is not strictly imprisoned to the miniature-upon-the-miniature imitation of impulses, Homo sapiens is more prominently a pathological imitator that is likely the consequence of recurring experience to define and adopt behavior by habit that is oriented to dampen, not pathologically caused death, but accustomed and unperceived motions received from the external, entangled to it, it is embodied to life experience. Rare single, events of the pastt might be postulated to explain the presence of ascetic behavior, but are hard to account for the temporal propagation of self-defeating behavior unless they also entail enduring motions.

Stores are filled with long-acting products to kill pain, extended warranties on devices, automobiles, technological advances make available for the citizen that were once believed to be to expensive to be possible, nothing seems to last but incentive to effect lasting goals . A futile situation has presented itself whereupon the citizen is made aware that the environment itself is as potentially labile as lifespan. Paradox ridden concepts are evolved and propagated to contain a smallened but long acting horizon, redeemed for material technological produce that is prominent throughout daily life. Whether suffering from either natural or man-made disaster, it is perhaps painful to consider that men have created their own survival bubble that is realized in physical form to a drift/diffuse-away island from the actual. Though it may be conceptually difficult enough to conceive a a real nature to divisions between pathological and natural, it is possibly also the case that a distant surface, causing pathological motions to the ecosphere, drifts like a bubble in space to resemble the free floating geometries employed in conceptual constructions; entangled with the self and environment false concepts have a strong seductive hold on mind and body. It is potentially fatefully the case that a search for new scientific revelations exclude a wholesome perspective that excludes the consideration of physical facets that are external to the witnessible grasp in their hold upon the familiar elements of the common niche, preclude serendipities entangled to their probability of discovery. It is suggested that contemporary study is re-oriented with an open imagination to elaborate a manifestation of the concept mass as it is ensued from paradox referenced by number as it, interfaces description bearing nominals, to find a perspective that has spaces for the unsuspected unknown, is 
appropriate to steer seeking to achieve a positive realignment of instinct in researches and behaviors. 


\section{Conclusion}

Tangible ideations of an elsewhere, contained to rationalized form are falsely considered a viable mode to physically bridge un-spanned or unspannable physical divides. 'A teleportation of the elaboration' in a mechanism of exploration seeking to resolve a chronic pathological suffering, is given to describe consequences realized to the meeting of individuals and environments with unperceived motions indigenous to an unknown surface.

\section{References}

Bub, Jeffery, Quantum Entanglement and Information, in Stanford Encyclopedia of Philosophy, 2010. http://plato.stanford.edu/entries/qt-entangle/.

Brown, Bill, Thing Theory, Critical Inquiry, Volume 28, Number 1, pgs. 1-12, 2001. http://faculty.virginia.edu/theorygroup/docs/brown.thing-theory.2001.pdf

Cantor, Matt, Think Hard: Telekinetic Toys On Their Way Concentration 'Magically' Lifts Ball in Tube, Newser, Apr 23,2009.http://www.newser.com/story/56986/think-hard-telekinetic-toys-on-theirway.html.

Connor, Steve,The Big Question: Is time travel possible, and is there any chance that it will ever take place? The Independent, London, U.K. Feb, 2008.

http://www.independent.co.uk/news/science/the-big-question-is-time-travel-possible-and-isthere-any-chance-that-it-will-ever-take-place-779761.html

DeNoon, Daniel, AIDS/Smallpox Vaccine OK in Early Test AIDS Vaccine That Protects Monkeys Looks Good in First Human Test Copyright 2007, WebMD Inc http://www.cbsnews.com/stories/2007/02/09/health/webmd/main2457822.shtml.

de Sitter, W., On Distance, Magnitude, and Related Quantities in an Expanding Universe Bulletin of the Astronomical Institutes of the Netherlands, Vol. 7, p.205, 1934.

Einstein, Albert, Relativity: Chpt. XI The Lorenz Transformations, in The Special and General Theory, (R.W. Lawson transl), NewYork,: Bartleby.com, 2000.

http://www.bartleby.com/173/11.html.

Field, J.V Kepler's Geometrical Cosmology, University Of Chicago Press; $1^{\text {st }}$ edition, 1988. ISBN-13: 978-0226248233.

Foucault, Michel, Discipline and Punishment Vintage Books New York 1977. 
Fox News, Israeli Scientist Figures Out How Time Machine Might Work, Aug 2007.

http://www.foxnews.com/story/0,2933,293937,00.html

Halverson, Mellissa Sue, During the Smallpox Epidemics: an Evolution Native American Beliefs and Medical Treatments, 2007.

http://www.earlyamerica.com/review/2007_summer_fall/native-americans-smallpox.html.

Kirsh, Marvin, E., Anthropology and Parallelism : The Individual as a Universal, International Journal of Sociology and Anthropology 1(7):112-115, 2009.

http://www.academicjournals.org/ijsa/PDF/Pdf2009/November/Kirsh.pdf

Kirsh, Marvin, E., Evolution at the Surface of Euclid:Elements of a Long Infinity in Motion Along Space, International Journal of the Arts and sciences 4(2):71-96, 2011 a. http://ssrn.com/abstract $=1575622$

Kirsh, Marvin, E. Science, the Virtual and the Actual: A Real Stand-off, (forthcoming), International Journal of Science in Society, 2011b.

Malinowski, Bronislaw, The Subject Method and Scope of this Inquiry, in Erickson, Paul, A. and Murphy, Liam, D. (2006) Readings for a History of Anthropological Theory 2nded., edition Broadview Press Ontario Canada, pps. 171-186, 1972.

Martinez-Conde S, The Lab Route to Out-of-Body Experiences, Mind Matters, the Scientific American web seminar on mind and brain., Jan 2008, http://smc.neuralcorrelate.com/files/publications/martinez-conde_mindmatters08.pdf

Martinez-Conde S Mind Matters, Blindsight: When the brain sees what you do not, Mind Matters The Scientific American blog on science and mind. April 2007, http://smc.neuralcorrelate.com/files/publications/martinez-conde_mindmatters07a.pdf

Nietzsche F. On the Genealogy of Morals. trans. Walter Kaufmann and R.J. Hollingdale, in On the Genealogy of Morals and Ecce Homo. New York: Random House, 1967.

Ouellette, Jennifer, Gravity Probe B Says Einstein was Right. Again. Discovery News, May 5, 2011. http://news.discovery.com/space/gravity-probe-b-110506.html.

Popescu, S., and Rohrlich,D., The Joy of Entanglement, in. Introduction in quantum information and computation, (H-K Lo, S Popescu and T Spiller, eds )World Scientific, 1998.

Poster, Carol, Protagoras (fl. 5th C. BCE) in Internet Encyclopedia of Philosophy (Fieser, James, Dowden,Bradley eds.) (april 27 2005). http://www.iep.utm.edu/protagor/ 
Pravda, Time Machine Built in Europe, Russian Scientists Say, June, 2010.

http://english.pravda.ru/science/tech/06-08-2010/114515-time_machine-0/

Raymond S Weinstein, Michael M Weinstein, Kenneth Alibek, Michael I Bukrinsky, Beda Brichacek, Significantly reduced CCR5-tropic HIV-1 replication in vitro in cells from subjects previously immunized with Vaccinia Virus, BMC Immunology 2010, 11:23 (18 May 2010).

Schrödinger, E. (1935) "Discussion of Probability Relations Between Separated Systems,," Proceedings of the Cambridge Philosophical Society, 31: 555-563; 32 (1936): 446-451.

Spicer, Jack "Radar" from My Vocabulary Did This To Me: The Collected Poems of Jack Spicer. Middletown: Wesleyan University Press, 2008.

http://www.poetryfoundation.org/poem/182427.

Tilton, Homer, Smarandache, Florentin, (eds) Todays Take on Einsteins' Relativity Proceeding of the Conference at Pima Community College ISBN 1-932-233-24-1,Pima College Press, Feb 8 2005. Preface page 5 .

Yoshida, J., H. Saitoh, Z, Confinement of electron plasma by levitating dipole magnet, Phys.

Plasmas 17, 2010.

Zhukhovitskii D.I., Levitation of Atoms over a Metal Surface Russ. J. Phys. Chem., vol. 80, no. 9, pp. 1489-1497, 2006, http://oivtran.ru/dmr/index.html. 